\title{
Reduced gray matter volume of left superior temporal gyrus in schizophrenia with auditory verbal hallucinations: a voxel- based morphometry study
}

\author{
Huawang $\mathrm{Wu}^{1,2}$, Fengchun $\mathrm{Wu}^{2}$, Xiaoyin $\mathrm{Ke}^{2}$, Ripeng $\mathrm{Li}^{2}$, Xiaobing $\mathrm{Lu}^{2}$ and Yuping $\mathrm{Ning}^{2, \mathrm{a}}$ \\ ${ }^{1}$ Key Laboratory for Neurolnformation of Ministry of Education, School of Life Science and Technology, University of \\ Electronic Science and Technology of China, Chengdu, 625014, China \\ ${ }^{2}$ The Affiliated Brain Hospital of Guangzhou Medical University (Guangzhou Huiai Hospital), Guangzhou, 510370, \\ China
}

\begin{abstract}
Auditory verbal hallucinations (AVHs) which are the core psychopathology symptoms in schizophrenia patients cause amounts of distress, functional disability and behavioral dys-control. However, the neural mechanism with the onset of AVHs in schizophrenia remains unclear. In the present study, we recruited 30 schizophrenia patients with AVHs, 20 schizophrenia patients with non-AVHs (nAVHs), and 50 healthy controls. Structure magnetic resonance imaging was obtained, and voxel-based morphometry (VBM) was used to analyse the gray matter volume (GMV) in the three groups. We found that the GMV of anterior cingulate cortex (ACC), left superior temporal gyrus (STG), and left middle temporal gyrus (MTG) significantly decreased in schizophrenia patients compared to healthy controls. In addition, the left STG with significantly decreased volume was found in nAVHs compared to AVHs, but no significant changes of GMV in ACC and left MTG were found between AVHs and nAVHs. Our current study identified the abnormal GMV of the left STG in schizophrenia patients with AVHs. Our findings further supported the view that, whilst mild reduced GMV of the left STG might still enable the emergence of AVHs, more severe alterations may prevent the occurrence of AVHs experience.
\end{abstract}

\section{Introduction}

Auditory verbal hallucinations (AVHs) were typically defined as hearing a voice or other sound in the absence of an external stimulus. The life-time prevalence was about $7.25 \%$ in general population, and the age of the highest prevalence rate was less than 30 years and $1.1 \%$ of individual sought professional help for AVHs[1]. AVHs which was the core psychopathology symptom affected about $60-80 \%$ schizophrenia patients and caused amounts of distress, functional disability and behavioral dys-control[2,3].

Structural magnetic resonance imaging has begun to provide insights into the neuroanatomy of AVHs in schizophrenia. A meta-analysis revealed that the severity of AVHs was associated with gray

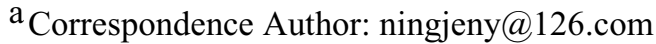

This work was supported by the National Basic Research Program of China (973 program, 2011CB707801).
} 
matter volume (GMV) reductions in left superior temporal gyrus (STG) but without significance changed of GMV between AVHs and nAVHs group[4]. Another study showed that the volume of the putamen was lower in AVHs compared to nAVHs and no other brain regions with significantly changed GMV were found between the two groups [5]. A recent study of AVHs found that decreased bilateral thalamic GMV was associated with the severity of AVHs [6]. These findings indicated that the neuroanatomical mechanism of AVHs remains controversial.

In the current study, we recruited 30 schizophrenia patients with AVHs and 20 schizophrenia patients without history of AVHs and 50 aged, gender matched healthy controls. The two patients groups were well matched in clinical variables and without combined any other hallucinations except for AVHs. We employed the voxel-based morphology (VBM) to evaluate the relationships between the GMV and AVHs symptom.

\section{Methods}

\subsection{Participants}

In this study, 50 schizophrenia subjects and 50 healthy controls were recruited at the Affiliated Brain Hospital of Guangzhou Medical University and all were right-handed and Han Chinese ancestry with the age between 18 and 45 ages. Schizophrenia patients were assessed by two experienced psychiatrist and diagnoses with schizophrenia using the structured clinical interviewer of DSM-IV (SCID) Patient Edition. The Positive and Negative Syndrome Scale (PANSS) was used to evaluate clinical symptoms of psychosis. A score of 4 points or more must be at least three items among the positive symptoms of PANSS and the total scores of PANSS were at least of 60 points. According to the experience of AVHs, schizophrenia patients were subdivided into two groups: AVHs group including patients who experienced AVHs in the past 24 hours with or without history of AVH, and nAVHs group including patients who had never experienced AVH during the course of illness before the MRI scanning. And all of schizophrenia patients were never experienced any other hallucinations including visual hallucination, olfaction hallucination, tactile hallucination, gustatory hallucination, visceral hallucination and motor hallucination. The Auditory Hallucination Rating Scale was used to assess AVHs on seven characteristics: frequency, reality, loudness, number of voices, length, attention dedicated to hallucinations, and hallucination-induced arousal. All healthy controls were screened using the SCID Non-Patient Edition to confirm the lifetime absence of Axis I illness and selected control subjects had no known history of psychiatric illness in the any two lines of first-to third degree biological relatives. All schizophrenia patients and healthy controls were without a lifetime history of seizures, head trauma, serious medical or surgical illness, substance abuse or dependence, or contraindications for MRI. Potential participants were excluded if discovered gross abnormalities signals on the cerebral. The written informed consent was obtained from all subjects and approved by the local ethics committees of the Affiliated Brain Hospital of Guangzhou Medical University.

\subsection{Data acquisition}

MRI data was acquired on a 3.0-Tesla MR imaging system (Achieva X-series, Philips Medical Systems, Best, the Netherlands) with an eight-channel SENSE head coil, in the Department of Radiology, the Affiliated Brain Hospital of Guangzhou Medical University, Guangzhou, China. Whole brain structural images were acquired with a three dimensional T1-weighted turbo field-echo (TFE) sequence. The following parameters were used during the scanning: repetition time (TR)= $8.2 \mathrm{~ms}$; echo time $(\mathrm{TE})=3.7 \mathrm{~ms}$; flip angle $(\mathrm{FA})=7^{0} ; \mathrm{FOV}=256 \times 256 \mathrm{~mm}^{2} ; 256 \times 256$ acquisition matrix; $1 \mathrm{~mm}$ slice thickness without inter-slice gap; voxels size $=1 \times 1 \times 1 \mathrm{~mm}^{3}$, and 188 continuous sagittal slices. 


\subsection{Data Analysis}

All the 3D T1 weighted images were performed by using VBM8 toolbox (http://dbm.neuro.unijena.de/vbm8) for SPM8 package (http://www.fil.ion.ucl.ac.uk/spm). The default procedures in the VBM8 toolbox were used for preprocessing. All T1 Images were corrected for bias-field inhomogeneity; segmented into GM, white matter and cerebral spinal fluid; and normalized to standard space using high dimensional DARTEL normalization. The normalized GM images were then modulated account for volume changes resulting from the normalization process. The normalized and modulated images were smoothed with $8 \mathrm{~mm}$ full-width at half-maximum (FWHM) smoothing kernel for final statistical analysis. After this preprocessing, we obtained smoothed, modulated, normalized data that were used for the statistical analysis. To achieve maximal sensitivity, to optimize voxel residual smoothness estimation and to exclude false positives in non-GM tissue, voxel-wise comparisons were masked using a comparison specific explicit optimal threshold gray matter mask created using the Masking toolbox (www.cs.ucl.ac.uk/staff/g.ridgway/masking).

\subsection{Statistical Analysis}

Statistical group difference maps were constructed using a general linear model (GLM) by performing ANOVA to test for the main effect of group with sex and age as covariates. The threshold for voxelwise comparisons of GMV was set at $p<0.05$, family-wise error (FWE)-corrected at the cluster level (corrected for non-stationarity of smoothness for VBM data) with an initial voxel threshold of $p<$ 0.001 . We then extracted the mean GMV of clusters showing significant changes in main effect of group and then exported these to SPSS for post-hoc analysis with Bonferroni corrected $(p<0.05)$. A correlation analysis between the mean GMV of clusters with clinical variables in schizophrenia patients with AVHs also performed.

\section{Results}

\subsection{Demographic and Clinical Characteristics}

Demographic and clinical data for the subjects are shown in Table 1. The three groups were wellmatched in gender, age and years of education. There were no significant differences in durations of illness, PANSS positive score, PANSS negative score, PANSS general score and PANSS total score between the two schizophrenia patients groups.

Table 1. Demographic information for schizophrenia patients and healthy controls.

\begin{tabular}{lllll}
\hline Characteristics & AVHs(n=30) & nAVHs $(\mathbf{n}=\mathbf{2 0})$ & HC(n=50) & $\boldsymbol{P}$-value \\
\hline Age(years) & $27.30 \pm 5.27$ & $30.5 \pm 6.77$ & $28.56 \pm 6.35$ & 0.191 \\
Sex(male/female) & $18 / 12$ & $14 / 6$ & $34 / 16$ & 0.700 \\
Education(years) & $11.70 \pm 3.29$ & $10.65 \pm 3.08$ & $11.94 \pm 2.71$ & 0.260 \\
Duration of illness(months) & $45.97 \pm 56.26$ & $54.25 \pm 45.80$ & - & 0.586 \\
PANSS & & & & \\
Positive subscale & $25.10 \pm 5.23$ & $23.95 \pm 3.41$ & - & 0.390 \\
$\quad$ Negative subscale & $22.67 \pm 8.20$ & $22.25 \pm 6.10$ & - & 0.847 \\
$\quad$ General psychopathology & $39.80 \pm 9.67$ & $42.55 \pm 7.90$ & - & 0.296 \\
$\quad$ Total score & $87.57 \pm 19.27$ & $88.75 \pm 10.52$ & - & 0.803 \\
AHRS total scores & $28.23 \pm 5.22$ & - & - & - \\
\hline
\end{tabular}




\subsection{Group differences in GMV}

A main effect of group was observed in the anterior cingulate cortex (ACC), the left superior temporal gyrus (STG) and the left middle temporal gyrus (MTG). Compared to healthy controls, the schizophrenia patients with or without AVHs showed lower GMV of the ACC, the left STG and the left MTG, and significant lower GMV in left STG in nAVHs group than AVHs groups. However, no significant differences of GMV in ACC and left MTG was found between AVHs and nAVHs groups.

\subsection{Correlation between alterations in GMV and AVH symptom severity}

We don't find any correction between alterations in GMV and AHRS total scores in AVHs group.

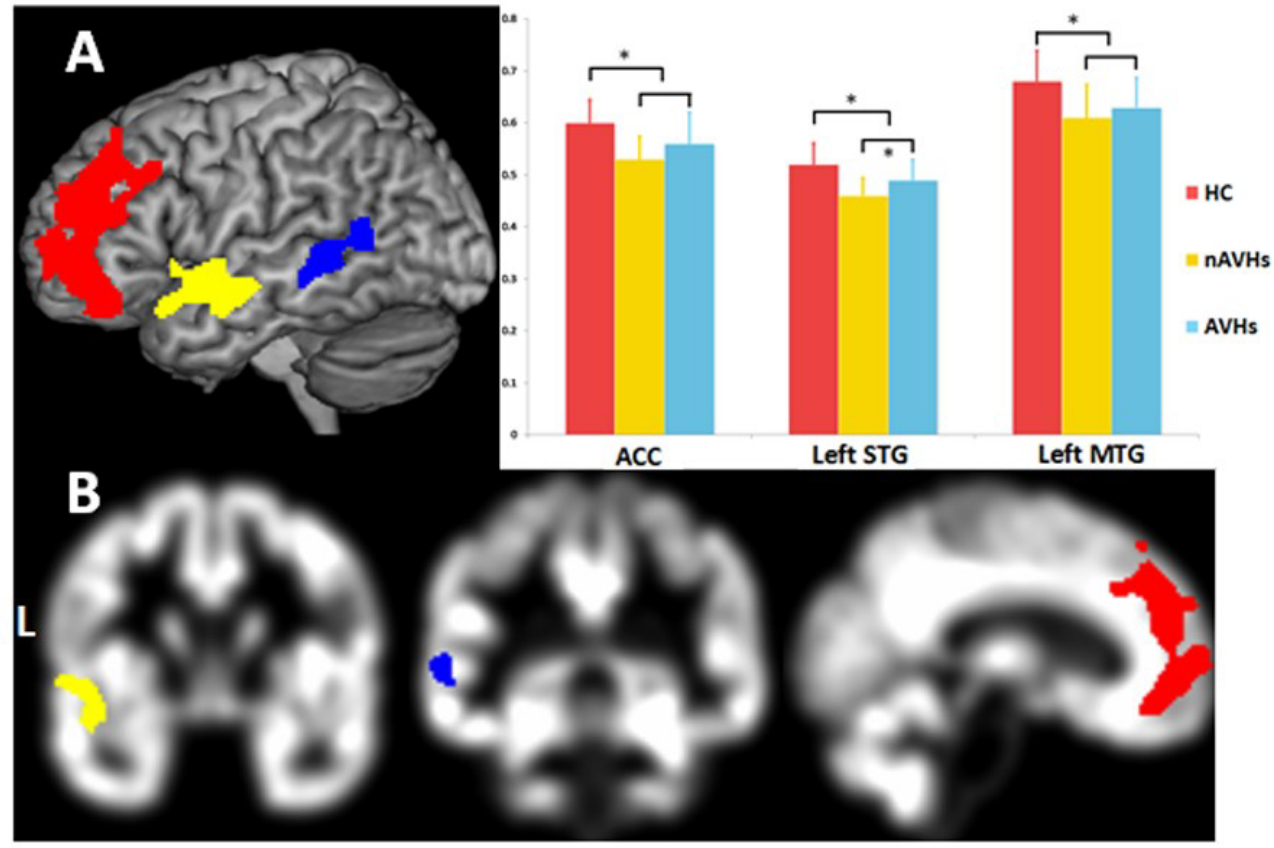

Figure 1. Changed GMV in schizophrenia patients with AVHs. Main effects of mapping showed significant GMV differences in ACC, left STG and left MTG (The threshold was set at $p<0.05$, FWE corrected at the cluster level) (see A and B). The bar chart displayed different GMV of these clusters between three groups. * Significant difference between groups using post-hoc analysis with Bonferroni corrected, $P<0.05$.

\section{Conclusions}

In this study, we used VBM to evaluate the changes of GMV of schizophrenia with or without AVHs. We find that the GMV of the ACC, the left STG and the left MTG significantly decreased in schizophrenia patients compared to healthy controls. The AVHs group showed intermediate GMV in the STG compared to the nAVHs group and healthy controls, but no significantly changed of GMV in the ACC and left MTG between the AVHs and nAVHs groups. Our findings indicate that the left STG plays an important role in the neural underpinnings of AVHs in schizophrenia, and further support to the hypothesis that a certain degree of preserved the GMV of the left STG may contribute to the onset of AVHs in schizophrenia. 


\section{References}

1. B Krakvik, F Laroi, AM Kalhovde, K Hugdahl, K Kompus, O Salvesen, TC Stiles, E VedulKjelsas Scand J Psychol 16:12236 (2015).

2. TH Nayani, AS David Psychol Med 26:177-189 (1996).

3. SS Shergill, RM Murray, PK McGuire Schizophr Res 32:137-150 (1998).

4. G Modinos, SG Costafreda, MJ van Tol, PK McGuire, A Aleman, P Allen Cortex 49:1046-1055 (2013).

5. MJ van Tol, L van der Meer, R Bruggeman, G Modinos, H Knegtering, A Aleman Neuroimage Clin 4:249-257 (2013).

6. P Huang, Y Xi, ZL Lu, Y Chen, X Li, W Li, X Zhu, LB Cui, Q Tan, W Liu, C Li, D Miao, H Yin Scientific report(2015). 